\title{
Implications of liver injury in risk-stratification and management of patients with COVID-19
}

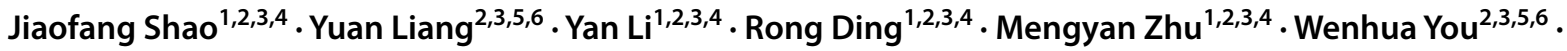 \\ Ziyu Wang ${ }^{1,2,3,4} \cdot$ Bin Huang ${ }^{1,2,3,4} \cdot$ Min Wu $\mathbf{W}^{1,2,3,4}$. Tingting Zhang ${ }^{1,2,3,4} \cdot$ Kening $\mathrm{Li}^{1,2,3,4}$. Wei $\mathrm{Wu}^{1,2,3,4}$.

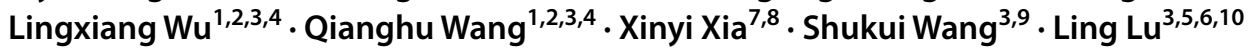

Received: 21 July 2020 / Accepted: 15 December 2020 / Published online: 6 February 2021

(c) Asian Pacific Association for the Study of the Liver 2021

\begin{abstract}
Background Infection with SARS-CoV-2 has been associated with liver dysfunction, aggravation of liver burden, and liver injury. This study aimed to assess the effects of liver injuries on the clinical outcomes of patients with COVID-19.

Methods A total of 1520 patients with severe or critical COVID-19 from Huoshenshan Hospital, Wuhan, were enrolled. Chronic liver disease (CLD) was confirmed by consensus diagnostic criteria. Laboratory test results were compared between different groups. scRNA-seq data and bulk gene expression profiles were used to identify cell types associated with liver injury.

Results A total of $10.98 \%$ of patients with severe or critical COVID-19 developed liver injury after admission that was associated with significantly higher rates of mortality $(21.74 \%, p<0.001)$ and intensive care unit admission $(26.71 \%, p<0.001)$. Pre-existing CLDs were not associated with a higher risk. However, fatty liver disease and cirrhosis were associated with higher risks, supported by evidences from single cell and bulk transcriptome analysis that showed more TMPRSS2 ${ }^{+}$cells in these tissues. By generating a model, we were able to predict the risk and severity of liver injury during hospitalization. Conclusion We demonstrate that liver injury occurring during therapy as well as pre-existing CLDs like fatty liver disease and cirrhosis in patients with COVID-19 is significantly associated with the severity of disease and mortality, but the presence of other CLD is not associated. We provide a risk-score model that can predict whether patients with COVID-19 will develop liver injury or proceed to higher-risk stages during subsequent hospitalizations.
\end{abstract}

Keywords Fatty liver disease $\cdot$ Viral hepatitis $\cdot$ Cirrhosis $\cdot$ Hospitalization $\cdot$ Disease progression $\cdot$ Prognosis $\cdot$ ICU admission · scRNA-seq analysis · TMPRSS2 Clinical prediction model

\begin{tabular}{|c|c|c|}
\hline & Abbrevia & ns \\
\hline & ACE2 & Angiotensin-converting enzyme 2 \\
\hline & ALP & Alkaline phosphatase \\
\hline & ALT & Alanine aminotransferase \\
\hline $\begin{array}{l}\text { Supplementary Intormation The online version contains } \\
\text { supplementary material available at https//doi org/10 1007/s1207 }\end{array}$ & AST & Aspartate aminotransferase \\
\hline 2-020-10123-0. & $\mathrm{LDH}$ & Lactate dehydrogenase \\
\hline & $\mathrm{CHB}$ & Chronic hepatitis B \\
\hline The first 4 names are first authors. & $\mathrm{CHC}$ & Chronic hepatitis $\mathrm{C}$ \\
\hline & $\mathrm{CI}$ & Confidence interval \\
\hline $\begin{array}{l}\text { Qianghu Wang } \\
\text { wangqh@njmu.edu.cn }\end{array}$ & CLD & Chronic liver disease \\
\hline & CRP & C-reactive protein \\
\hline $\begin{array}{l}\text { Xiny1 Xia } \\
\text { xinyixia@nju.edu.cn }\end{array}$ & FLD & Fatty liver disease \\
\hline Shukui Wang & GGT & $\gamma$-Glutamyl transpeptidase \\
\hline sk_wang@njmu.edu.cn & HBV & Hepatitis B virus \\
\hline & $\mathrm{HCV}$ & Hepatitis $\mathrm{C}$ virus \\
\hline $\begin{array}{l}\text { Ling Lu } \\
\text { lvling@ @jmu.edu.cn }\end{array}$ & MAFLD & $\begin{array}{l}\text { Metabolic dysfunction-associated fatty liver } \\
\text { disease }\end{array}$ \\
\hline
\end{tabular}


scRNA-seq Single-cell RNA sequencing

TMPRSS2 Transmembrane protease serine 2

\section{Introduction}

A novel coronavirus named SARS-CoV-2 began to rapidly spread across the world in December 2019 and was declared a global pandemic by the World Health Organization. The attachment of SARS-CoV-2 to the target cell is initiated by interactions between the spike glycoprotein (S) of the virus and its receptor of the host cell, angiotensin-converting enzyme 2 (ACE2). Subsequently, SARS-CoV-2 S protein is cleaved by a plasma membrane-associated type II transmembrane serine protease (TMPRSS2), leading to membrane fusion which is essential to release the viral contents into the infected cell cytosol. Both ACE2 and TMPRSS2 are essential for viral spreading to the host cell [1].

Several recent studies have shown that more than 50\% of patients with COVID-19 develop liver abnormalities, of whom $20 \%$ have liver injury [2-5]. Furthermore, a pathological study based on patients who had died from severe COVID-19 showed that their liver tissue had moderate microvesicular steatosis and mild lobular and portal activity indicating that SARS-CoV-2 might cause liver injury [6]. Previous research has also examined the association between markers of liver injury and mortality rates in patients with COVID-19, and has reported that aspartate transaminase (AST) levels display the highest correlation with mortality compared to other indicators of liver injury [7].

Chronic liver disease (CLD), such as chronic viral hepatitis, metabolic dysfunction-associated fatty liver disease (MAFLD) that was previously termed non-alcoholic fatty liver disease, and alcohol-related liver disease, affects approximately 1.5 billion people throughout the world and causes 2 million deaths each year [8]. Previous studies have shown that $2-11 \%$ of patients with COVID-19 have a preexisting CLD $[2,4,9]$. Recent studies have found that obese patients with MAFLD have higher risks of severe COVID19 symptoms [10]. However, research on early risk stratification and management is limited. Thus, the purpose of this study was to explore the implication of liver injury and CLD in patients with COVID-19.

\section{Methods}

\section{Study design and data collection}

We collected electronic health records, including medical history and all laboratory results, from February 4 to April 10 for 1520 patients diagnosed with severe or critical COVID-19 and admitted to Huoshenshan hospital from
February 4 to March 30, 2020, of which 1466 performed liver function tests. Written informed consent was waived due to the urgency of the COVID-19 pandemic. The diagnosis and severity of COVID-19 were based on practice guidelines issued by The Chinese National Health Commission (http://en.nhc.gov.cn/2020-03/29/c_78469.htm).

We used a six-category scale score to describe the clinical status of COVID-19: (1) discharged; (2) hospitalized, not requiring oxygen therapy; (3) hospitalized, requiring low-flow oxygen therapy; (4) hospitalized, requiring high-flow oxygen therapy, noninvasive mechanical ventilation, or both; (5) hospitalized, requiring extracorporeal membrane oxygenation, invasive mechanical ventilation, or both; (6) death. Higher scores indicated higher risks (Table 1).

\section{Liver injury definition and chronic liver disease classification}

The upper limit unit of normal of liver function tests was as follows: alanine aminotransferase (ALT), 40 IU/L; AST, $40 \mathrm{IU} / \mathrm{L}$; total bilirubin, $17.1 \mu \mathrm{mol} / \mathrm{L}$; total bile acid, $10 \mu \mathrm{mol} / \mathrm{L}$; gamma-glutamyl transferase (GGT), $50 \mathrm{IU} / \mathrm{L}$; and alkaline phosphatase (ALP), 125 IU/L. Patients whose ALT and/or AST, ALP, and/or GGT levels were higher than twice the upper limit unit of normal were considered as having hepatic injury [3], which were further classified by hepatocellular type (elevation of AST/ALT) and cholestatic type (elevation of ALP/GGT) (Table 2).

Based on the above criterion together with a categorization of when the abnormal liver function value occurred for the first-time relative to hospitalization time, the patients with liver injury were grouped as: a pre-admission injured group that had patients who had already presented with liver injury on admission, and post-admission injured group that had patients who developed liver injury during hospitalization.

Pre-existing CLDs, including chronic hepatitis B (CHB), chronic hepatitis $\mathrm{C}$ (CHC), and fatty liver disease (FLD), were diagnosed by consensus diagnostic criteria.

\section{Statistical analysis}

The statistical analyses in this study were performed using $\mathrm{R}$ (version 3.6.0). Fisher's exact test was applied for categorical variables. We utilized the Mann-Whitney $U$ test or Kruskal-Wallis $H$ test for continuous variables, and the results were presented as the median $(25 \%-75 \%$ interquartile range, IQR). A $p$ value $<0.05$ was considered statistically significant. 
Table 1 Characteristics of 121 COVID-19 patients with different types of chronic liver disease

\begin{tabular}{|c|c|c|c|c|c|}
\hline & Total $(n=121)$ & $\mathrm{CHB}(n=64)$ & $\mathrm{CHC}(n=20)$ & $\operatorname{FLD}(n=37)$ & $p$ value \\
\hline Age, median (IQR) & $62.00(51.00-69.00)$ & $63.00(54.75-70.00)$ & $66.50(58.75-70.25)$ & $57.00(38.00-66.00)$ & 0.015 \\
\hline Sex, $n(\%)$ & & & & & 0.163 \\
\hline Female & $43(35.54)$ & $22(34.38)$ & $4(20.00)$ & $17(45.95)$ & \\
\hline Male & $78(64.46)$ & $42(65.63)$ & $16(80.00)$ & $20(54.05)$ & \\
\hline \multicolumn{6}{|l|}{ Comorbidities, $n(\%)$} \\
\hline Hypertension & $33(27.27)$ & $15(23.43)$ & $7(35.00)$ & $11(29.73)$ & 0.506 \\
\hline Diabetes & $16(13.22)$ & $11(17.19)$ & $3(15.00)$ & $2(5.41)$ & 0.264 \\
\hline Cardiovascular disease & $10(8.26)$ & $5(7.81)$ & $2(10.00)$ & $3(8.11)$ & 0.908 \\
\hline Chronic nephrosis & $2(1.65)$ & $2(3.13)$ & $0(0.00)$ & $0(0.00)$ & 0.674 \\
\hline \multicolumn{6}{|l|}{ Treatment, $n(\%)$} \\
\hline Oxygen & $63(52.07)$ & $36(56.25)$ & $8(40.00)$ & $19(51.35)$ & 0.455 \\
\hline Convalescent plasma transfusion & $15(12.40)$ & $12(18.75)$ & $2(10.00)$ & $1(2.70)$ & 0.051 \\
\hline Antivirus & $103(85.12)$ & $52(81.25)$ & $19(95.00)$ & $32(86.49)$ & 0.354 \\
\hline Steroid & $18(14.88)$ & $11(17.19)$ & $1(5.00)$ & $6(16.22)$ & 0.502 \\
\hline \multicolumn{6}{|l|}{ Liver function tests } \\
\hline ALT (IU/L) & $28.75(18.15-46.18)$ & $27.10(15.69-43.00)$ & $25.29(14.88-46.19)$ & $36.70(24.69-61.47)$ & $\mathbf{0 . 0 3 8}$ \\
\hline $\mathrm{ALT}>40, n(\%)$ & $51(42.15)$ & $25(39.06)$ & $7(35.00)$ & $19(51.35)$ & 0.391 \\
\hline AST (IU/L) & $23.70(18.23-36.8)$ & $23.70(17.30-37.94)$ & $21.38(18.30-34.27)$ & $25.35(20.50-35.55)$ & 0.526 \\
\hline $\mathrm{AST}>40, n(\%)$ & $34(28.10)$ & 19 (29.69) & $4(20.00)$ & $11(29.73)$ & 0.734 \\
\hline TBIL $(\mu \mathrm{mol} / \mathrm{L})$ & $10.05(8.40-13.00)$ & $10.6(8.87-13.95)$ & $10.07(8.16-13.57)$ & $9.25(8.14-10.81)$ & 0.034 \\
\hline TBIL > 17.1, $n(\%)$ & $23(19.01)$ & $16(25.00)$ & $4(20.00)$ & $3(8.11)$ & 0.100 \\
\hline TBA $(\mu \mathrm{mol} / \mathrm{L})$ & $5.10(3.50-9.46)$ & $6.00(4.07-11.61)$ & $4.15(3.03-6.53)$ & $4.67(3.24-7.06)$ & 0.090 \\
\hline $\mathrm{TBA}>10, n(\%)$ & $41(33.88)$ & $28(43.75)$ & $4(20.00)$ & $9(24.32)$ & 0.052 \\
\hline $\operatorname{ALP}(\mathrm{IU} / \mathrm{L})$ & $73.70(59.70-91.93)$ & $71.05(57.38-82.58)$ & $83.90(65.75-105.35)$ & $73.09(61.59-100.39)$ & 0.146 \\
\hline $\mathrm{ALP}>125, \mathrm{n}(\%)$ & $15(12.40)$ & $7(34.38)$ & $2(20.00)$ & $6(16.22)$ & 0.749 \\
\hline GGT (IU/L) & $35.90(24.45-59.28)$ & $30.48(22.70-52.30)$ & $33.90(22.90-38.34)$ & $54.15(34.75-79.26)$ & $<0.001$ \\
\hline GGT $>50, n(\%)$ & $43(35.54)$ & $22(34.38)$ & $2(10.00)$ & $19(51.35)$ & 0.006 \\
\hline \multicolumn{6}{|l|}{ Laboratory parameters, median (IQR) } \\
\hline C-reactive protein $(\mathrm{mg} / \mathrm{L})$ & $35.9(24.45-59.28)$ & $30.48(22.70-52.30)$ & $33.90(22.90-38.34)$ & $54.15(34.75-79.26)$ & $<0.001$ \\
\hline IL-6 (pg/mL) & $2.98(1.50-14.250)$ & $3.46(1.50-16.38)$ & $2.35(1.64-3.85)$ & $2.75(1.50-6.85)$ & 0.550 \\
\hline D-dimer (mg/L) & $0.61(0.25-1.59)$ & $0.64(0.296-1.857)$ & $0.88(0.33-3.57)$ & $0.38(0.13-0.78)$ & 0.042 \\
\hline PT (s) & $93.90(90.00-97.45)$ & $93.80(89.61-96.50)$ & $91.60(87.60-96.30)$ & $96.90(93.58-100.99)$ & 0.003 \\
\hline INR & $0.61(0.25-1.59)$ & $0.64(0.30-1.86)$ & $0.88(0.33-3.57)$ & $0.38(0.13-0.78)$ & 0.042 \\
\hline LYM\# $\left(\times 10^{9 / L}\right)$ & $1.6(1.17-1.95)$ & $1.59(1.16-1.89)$ & $1.28(1.07-1.76)$ & $1.86(1.62-2.11)$ & 0.003 \\
\hline Clinical outcome, $n(\%)$ & & & & & 0.535 \\
\hline Discharged & $111(91.74)$ & $57(89.06)$ & $19(95.00)$ & $35(94.59)$ & \\
\hline Remained in hospital & $5(4.13)$ & $3(4.69)$ & $0(0.00)$ & $2(5.41)$ & \\
\hline Death & $5(4.13)$ & $4(6.25)$ & $1(5.00)$ & $0(0.00)$ & \\
\hline
\end{tabular}

\section{Liver sCRNA-seq data processing and estimation of the abundance of liver cell type}

The healthy liver and cirrhotic liver 10× scRNA-seq processed matrices were downloaded from GSE136103 [11], and the HBV-infected liver 10× scRNA-seq processed matrix was obtained from Ido Amit Lab [12]. The unsupervised clustering and visualization were performed in the Seurat R package v3.1.1 [13], and the liver progenitor clusters were determined by TROP2, ALB, AFP, KRT8,
KRT19, THY1, and KIT [14]. The cells expressing ACE2 and TMPRSS2 were counted, and Fisher's exact test was then conducted.

The bulk gene expression profiles from mixed cell types for HBV-infected, HCV-infected, MAFLD, cirrhotic, and healthy livers were downloaded from GSE83148, GSE149601, GSE130970, GSE112221, and GSE83148, respectively. CIBERSORTx, a web-based tool used for estimation of cell type abundances from bulk transcriptomes [15], was applied to estimate the abundance of 
Table 2 Clinical features of patients who had chronic liver disease with and without cirrhosis

\begin{tabular}{|c|c|c|c|c|}
\hline & Total $(n=127)$ & $\begin{array}{l}\text { Chronic liver disease with } \\
\text { cirrhosis }(n=13)\end{array}$ & $\begin{array}{l}\text { Chronic liver disease with- } \\
\text { out cirrhosis }(n=114)\end{array}$ & $p$ value \\
\hline Sex, $n(\%)$ & & & & 1.000 \\
\hline Male & $77(60.63)$ & $8(61.54)$ & $69(60.53)$ & \\
\hline Female & $50(39.37)$ & $5(38.46)$ & $45(39.47)$ & \\
\hline Age, median (IQR) & $63.00(51.50-69.00)$ & $68.00(60.00-74.00)$ & $62(51.25-68.75)$ & $\mathbf{0 . 0 3 8}$ \\
\hline Hospital stays, median (IQR) & $14.00(8.00-23.00)$ & $15.00(8.00-25.00)$ & $14.00(8.00-22.75)$ & 0.774 \\
\hline ICU admission, $n(\%)$ & $9(7.08)$ & $2(15.38)$ & $7(6.14)$ & 0.231 \\
\hline Highest six-category scale score & & & 0.045 & \\
\hline 2 & $58(45.67)$ & $2(15.38)$ & $56(49.12)$ & \\
\hline 3 & $45(35.43)$ & $6(46.15)$ & $39(34.21)$ & \\
\hline 4 & $18(14.17)$ & $5(38.46)$ & $13(11.40)$ & \\
\hline 5 & $1(0.79)$ & $0(0.00)$ & $1(0.87)$ & \\
\hline 6 & $5(3.94)$ & $0(0.00)$ & $5(4.39)$ & \\
\hline \multicolumn{5}{|c|}{ Laboratory parameters, median (IQR) } \\
\hline ALT (IU/L) & $28.75(18.15-46.17)$ & $25.49(14.90-34.40)$ & $29.62(18.30-47.73)$ & 0.318 \\
\hline AST (IU/L) & $23.70(18.23-36.8)$ & $29.50(17.10-45.40)$ & $22.90(18.38-35.55)$ & 0.342 \\
\hline $\operatorname{ALB}(g / L)$ & $37.70(34.3-40.9)$ & $33.15(32.00-34.50)$ & $38.1(35.22-40.96)$ & 0.002 \\
\hline GLB (g/L) & $28.00(25.00-30.50)$ & $32.48(29.04-34.41)$ & $27.7(24.70-29.85)$ & 0.006 \\
\hline TBIL $(\mu \mathrm{mol} / \mathrm{L})$ & $10.05(8.40-13.00)$ & $21.03(10.50-27.23)$ & $9.90(8.30-12.62)$ & 0.006 \\
\hline $\mathrm{DBIL}(\mu \mathrm{mol} / \mathrm{L})$ & $3.55(2.96-4.80)$ & $10.90(3.60-15.38)$ & $3.50(2.84-4.40)$ & 0.002 \\
\hline TBA $(\mu \mathrm{mol} / \mathrm{L})$ & $5.10(3.50-9.46)$ & $15.62(5.70-30.8)$ & $4.94(3.33-8.71)$ & 0.007 \\
\hline $\operatorname{ALP}(\mathrm{IU} / \mathrm{L})$ & 73.70 (59.70-91.92) & $91.92(77.60-116.33)$ & $71.10(58.60-89.84)$ & 0.005 \\
\hline GGT (IU/L) & $35.90(24.45-59.28)$ & $39.35(25.33-85.78)$ & $35.38(24.45-57.43)$ & 0.277 \\
\hline LDH (IU/L) & $186.65(164.75-244.06)$ & $255.2(193.42-267.97)$ & $184.10(162.55-229.98)$ & 0.011 \\
\hline MONO\% & $7.64(6.31-9.00)$ & $9.27(7.97-10.90)$ & $7.44(6.17-8.70)$ & $\mathbf{0 . 0 0 7}$ \\
\hline NEUT $\left(\times 10^{9 / \mathrm{L}}\right)$ & $3.62(2.92-5.28)$ & $2.98(2.31-3.28)$ & $3.80(2.93-5.35)$ & $\mathbf{0 . 0 3 3}$ \\
\hline $\operatorname{LYM}\left(\times 10^{9 / L}\right)$ & $1.60(1.18-1.95)$ & $1.06(0.87-1.17)$ & $1.69(1.26-1.98)$ & $<0.001$ \\
\hline CRP (mg/L) & $2.74(1.06-12.5)$ & $12.12(5.54-19.17)$ & $2.57(0.93-9.52)$ & 0.010 \\
\hline PT (s) & $13.19(12.49-14.04)$ & $15.68(14.36-17.08)$ & $13.10(12.44-13.88)$ & $<0.001$ \\
\hline D-Dimer (mg/L) & $0.61(0.24-1.59)$ & $3.57(1.97-5.04)$ & $0.52(0.22-1.30)$ & $<0.001$ \\
\hline IL-6 (pg/mL) & $2.98(1.50-14.25)$ & $22.78(9.86-27.84)$ & $2.70(1.50-6.62)$ & 0.006 \\
\hline
\end{tabular}

$T R O P 2^{+} T_{M P R S S}{ }^{+}$cells in different livers, using custom signature matrix extracted from scRNA-seq profile and mixture files from bulk RNA-seq as input, and the fractions of $T R O P 2^{+} T_{M P R S S}{ }^{+}$cells in each sample were downloaded for visualization and comparison.

\section{Construction of risk score}

The significance of each variable was assessed between the non-injury and post-admission injured groups by univariate logistic regression. Indicators with an odds ratio $(\mathrm{OR})>1$ and $p$ value $<0.001$ were used for the final model to investigate whether the patient would develop liver injury. We also selected significant indicators between patients who stayed at 2-4 scales and who developed into 5-6 scales to predict the highest six-category scale score. The performance of the scoring model was assessed using receiver operating characteristic (ROC) curves created from fivefold crossvalidation. The average area under ROC (AUROC) was calculated by the cvAUC R package (version 1.1.0).

\section{Results}

\section{Liver injury is associated with a poor prognosis in patients with COVID-19}

We identified 263 (17.9\%) patients with liver injury (Table S1) to explore the impact of liver injury on severe or critical cases of COVID-19. Amongst these patients, 102 (38.78\%) had presented with liver injury on admission (preadmission injured group) and 161 (61.22\%) patients developed liver injury during their hospitalization (post-admission injured group). As shown in Fig. 1a, hypertension was 

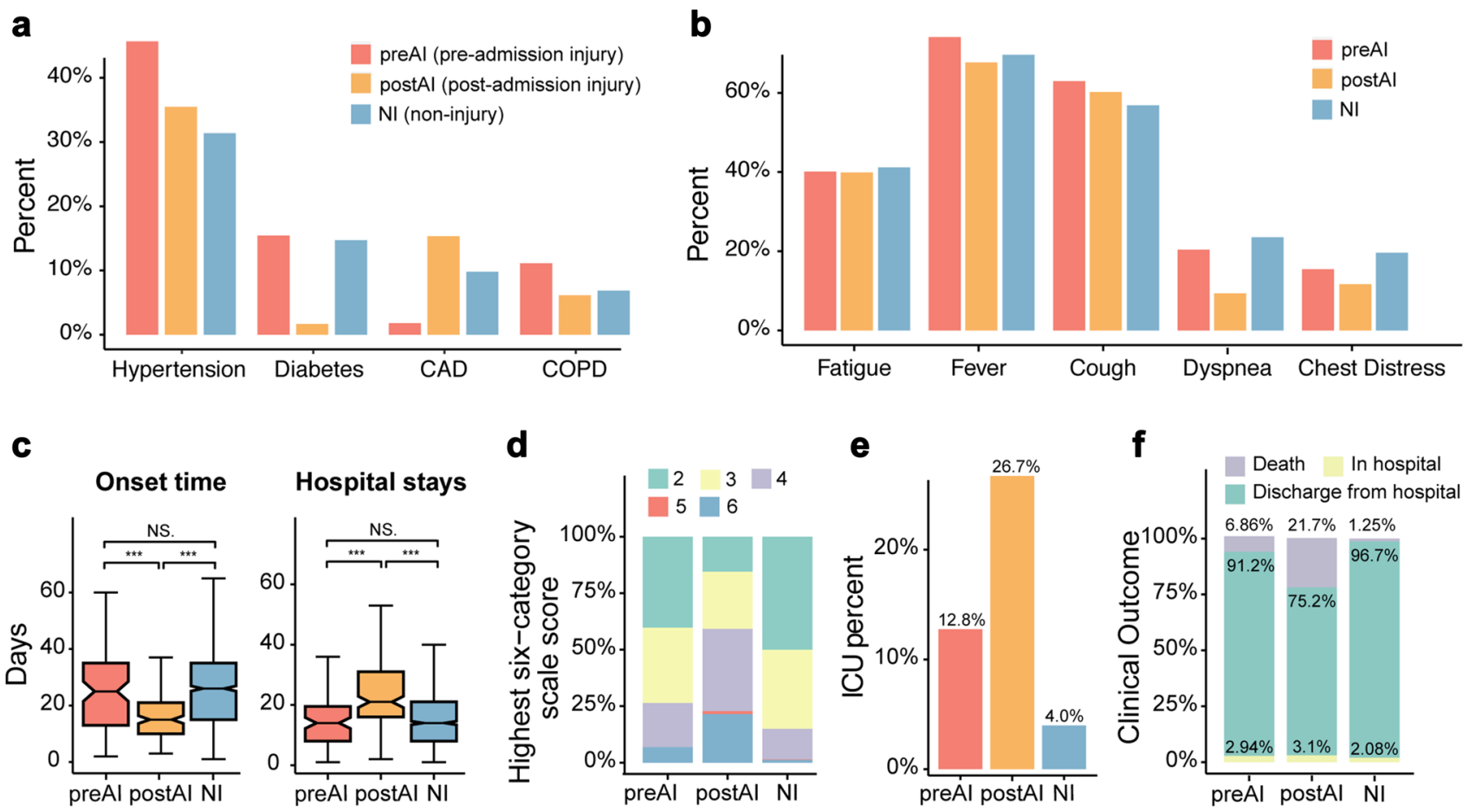

Fig. 1 Characteristics of patients with or without liver injury during hospitalization. a Common comorbidities. b Common COVID-19 symptoms. c Days from COVID-19 symptom onset to hospitalization and hospital stays. d Highest six-category scale scores during hospitalization. e ICU admission during hospitalization. f Final clinical outcome for patients in the three groups. The $y$-axis is percent-

more commonly seen in the post-admission injured group $(45.68 \%, p=0.025)$. The median time from symptom onset to admission was significantly shorter in the post-admission injured group than that in the pre-admission injured and non-injured groups (Fig. 1c, Fig. S1D, median: 15 vs. 25 or 26 days, $p<0.001$ ), suggesting that disease progression was faster in the post-admission injured patients. The length of hospital stay was significantly longer in the post-admission injured group (Fig. 1c, Fig. S1E, median 21 days) than that in the other two groups (median 14 days for each). Furthermore, the six-category scale scores for the post-admission injured group were significantly enriched in the 3-6 range (Fig. 1d), indicating a higher risk. Conversely, over $50 \%$ of patients without liver injury remained at levels 2 and 3 . The post-admission injured group also had significantly higher mortality rates than the pre-admission injured and noninjured groups during hospitalization (Fig. 1f, $21.74 \%$ vs. $6.86 \%$ or $1.25 \%, p<0.001$ ), as well as increased intensive care unit (ICU) admission rates (Fig. 1e, $26.71 \%$ vs. $12.75 \%$ or $3.99 \%, p<0.001)$.

There was no significant difference in age, sex and treatment strategy among the three groups (Fig. S1A, Fig. S1B, Fig. S1C). Among the 161 COVID-19 patients with liver injury during hospitalization, 59 were hepatocellular type age of patients with the corresponding characteristics out of the total patients in that groups. preAI pre-admission injury, liver injury identified upon admission, postAI post-admission injury, liver injury identified after admission, $N I$ non-injury, liver injury not found during the disease course

(elevation of AST/ALT), 61 cholestatic type (elevation of ALP/GGT), and 41 mixed type (Fig. S2A), but there was no significant difference in prognosis (Fig. S2B, Fig. S2C) and treatments (Fig. S2D).

\section{CLD is not significantly associated with a poor prognosis in patients with COVID-19}

We compared the differences between severe or critical COVID-19 patients with and without CLD to evaluate the influence of SARS-CoV-2 on patients with pre-existing CLD. As shown in Table S2, 127 (8.35\%) of the 1520 patients with severe or critical cases of COVID-19 had CLD, including 64 patients with $\mathrm{CHB}, 20$ with $\mathrm{CHC}, 37$ with FLD, and 6 with liver cirrhosis but without documented etiological factors. Among all the comorbidities tested in this study, hypertension was the only one that showed a significant difference between the groups $(27.56 \%$ with CLD vs. $37.19 \%$ without CLD, $p=0.034$ ).

Laboratory test results were also compared between the two groups. The median platelet count was significantly lower in the patients with CLD than that in those without CLD (206.00 $\times 10^{9 / \mathrm{L}}$ vs. $\left.220.00 \times 10^{9 / \mathrm{L}}, p=0.008\right)$. Interferon gamma was significantly decreased in patients with pre-existing CLD 
a
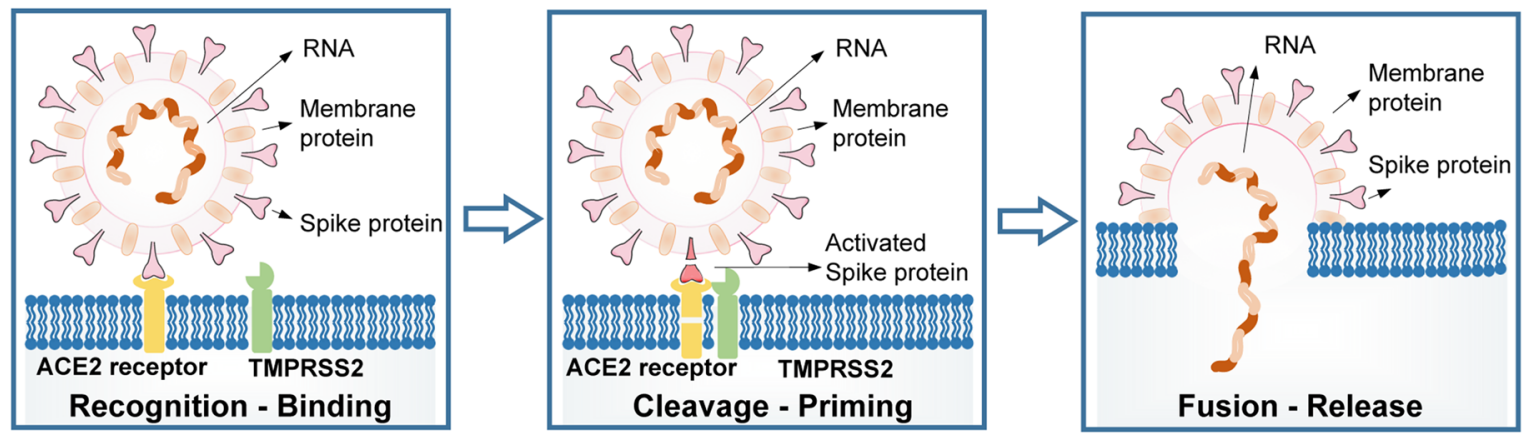

b
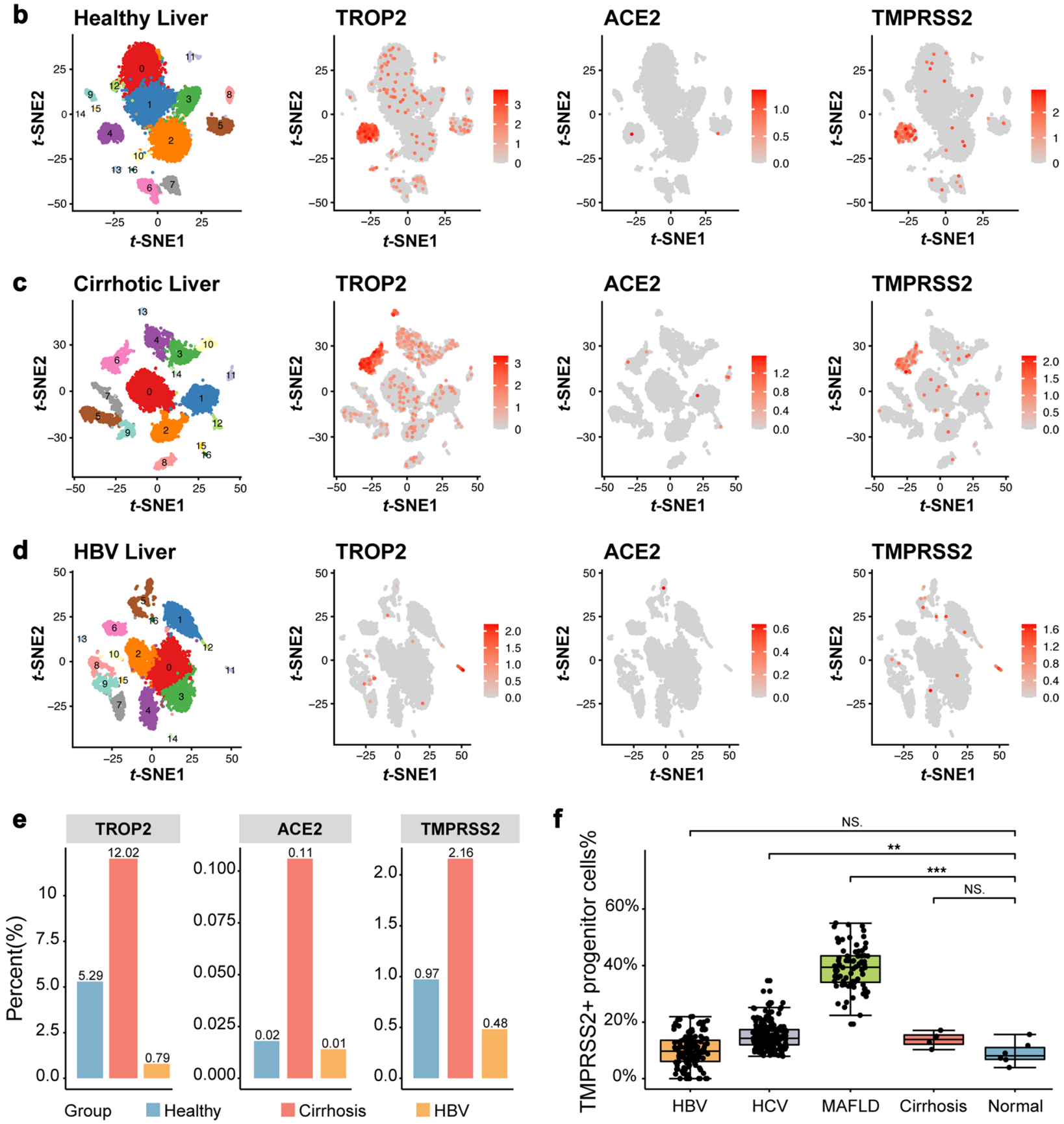
4Fig. 2 TMPRSS2 ${ }^{+}$cells fraction in different liver tissues. a Schematic of SARS-CoV-2 virus infection process. SARS-CoV-2 utilizes ACE2 for entry into cells and TMPRSS2 for spike (S) protein priming. Following membrane fusion, the virus RNA will be released into the host cell. tSNE representation of scRNA-seq expression matrix and expression maps for genes TROP2, ACE2 and TMPRSS2 in $\mathbf{b}$ healthy liver ( $n=11,106$ cells), c cirrhotic liver ( $n=6620$ cells), d HBV liver ( $n=7244$ cells). Color bars indicate $\log 2$ normalized expression. e Fractions of cells expressing TROP2, ACE2 and TMPRSS2 in the three liver scRNA-seq datasets. f Fractions of TROP2 $2^{+} T M P R S S 2^{+}$ cells in the bulk datasets from HBV, HCV, MAFLD, cirrhotic and healthy livers

than that in those without pre-existing CLD (median: 2.68 vs. $5.32 \mathrm{pg} / \mathrm{mL}, p=0.032$ ). A similar trend was also observed for interleukin- 2 and $\mathrm{CD}^{+} / \mathrm{CD}^{+}$T-helper cell fractions, but these changes were not statistically significant.

No significant evidence of CLD being a risk factor for the severity or mortality of COVID-19 was found. This result may be due to the consistent and targeted delivery of liver protection treatments in patients with CLD. In addition, this result implies that liver injury occurring during the course of COVID-19 is associated with a poorer prognosis but preexisting CLD is not.

\section{Patients with FLD are at a higher risk of liver injury compared to patients with viral hepatitis}

We conducted a comprehensive analysis of 121 patients with both COVID-19 and chronic liver comorbidities. Of these 121 patients, 64 (52.89\%) had CHB, 20 (16.53\%) had CHC, and 37 (30.58\%) had FLD (Table S3). The clinical outcomes were not significantly different among the different types of CLD $(p=0.535)$. However, all 5 recorded deaths occurred in patients with viral hepatitis. Patients with FLD had higher levels of ALT (median: $36.70 \mathrm{IU} / \mathrm{L}, p=0.038$ ) and GGT (median: $54.15 \mathrm{IU} / \mathrm{L}, p<0.001$ ) than those with CHB or CHC. Furthermore, over $50 \%$ of patients with FLD had abnormal levels of ALT $(p=0.391)$ and GGT $(p=0.006)$.

C-reactive protein (CRP) (median: $54.15 \mathrm{mg} / \mathrm{L}, p<0.001$ ) and the absolute lymphocyte count (lymphocyte\#; median: $1.86, p=0.003$ ) were higher in the FLD patients. Prothrombin time was also significantly prolonged (median: $96.90 \mathrm{~s}$, $p=0.003$ ) and the international normalized ratio was significantly lower in patients with FLD (median: 0.38, $p=0.042$ ), suggesting that coagulation disorders and dysfunction of the liver occurred concurrently in patients with pre-existing FLD. These results imply that patients with FLD suffer more severe liver damage.

\section{Patients with both COVID-19 and cirrhosis are at a higher risk of disease progression}

Cirrhosis is a complication of many liver diseases. Therefore, we analyzed the clinical characteristics and laboratory features of patients with CLD and with and without cirrhosis. As shown in Table S4, 13 (10.24\%) patients had CLD with cirrhosis, of which 4 had CHB, 2 had CHC, 1 had MAFLD, and 6 had cryptogenic cirrhosis. No significant differences in terms of hospital stays $(p=0.774)$ or ICU admission rates $(p=0.231)$ were observed. However, the highest six-category scale scores for patients with both CLD and cirrhosis were significantly enriched at 3 and 4 , while for those without cirrhosis were mainly at 2 and $3(p=0.045)$. This result indicated that patients with both CLD and cirrhosis were at a higher risk of disease progression.

We confirmed by examining laboratory results that most liver enzymes were significantly higher in patients with cirrhosis, except ALT, AST, and GGT. Moreover, the levels of D-dimer and two well-known pro-inflammatory biomarkers (interleukin-6 and CRP) were found to be higher in patients with cirrhosis. All evidence mentioned above showed that patients with both COVID-19 and cirrhosis were at an elevated risk of disease progression compared with the patients who had CLD without cirrhosis.

\section{Cirrhotic and fatty livers generate more TMPRSS2 ${ }^{+}$ cells}

We studied the liver scRNA-seq data from recent publications to investigate why patients with cirrhosis are more affected by SARS-CoV-2 [11, 12]. ACE2 and TMPRSS2 were shown to be necessary for the virus entry and infection (Fig. 2a). Consistent with many recent reports, the level of the SARS-CoV-2 entry-receptor $A C E 2$ was low in liver tissue. However, a small population of $T R O P 2^{+}$liver epithelial progenitors expressed ACE2 and TMPRSS2 (Fig. 2b-e). Of the 11,106 cells detected in healthy livers, only 2 cells expressed ACE2 and 108 expressed TMPRSS2 (Fig. 2b). Of the 6620 cells analyzed from cirrhotic livers, 7 cells expressed ACE2 and 143 expressed TMPRSS2 (Fig. 2c). This result represents a significant increase in the number of TMPRSS2 ${ }^{+}$cells in the cirrhotic livers ( $p<0.001$, Fisher's exact test). Of 7244 cells analyzed from untreated HBVinfected livers, only 1 cell expressed $A C E 2$ and 35 expressed TMPRSS2 (Fig. 2d). The TMPRSS2 ${ }^{+}$cells were significantly fewer in HBV-infected liver than those in both healthy and cirrhotic livers ( $p<0.001$, Fisher's exact test) (Fig. 2e).

We estimated the abundance of SARS-CoV-2 vulnerable cells for more liver bulk expression profiles with CIBERSORTx using the signatures built from the same healthy liver scRNA-seq dataset. To obtain a better reference signature, we limited TROP2 ${ }^{+}$TMPRSS $^{+}$cells to a subset of the cell population in "cluster 4" marked by progenitor markers ALB, KRT8 and KRT19 (Fig. S3). As ACE2 expression was too low to be confidently identified with general scRNAseq depth, we did not restrict the signature to $A C E 2^{+}$cells. As shown in Fig. S4, CIBERSORTx was run for each bulk 
transcriptome from different patients to impute TMPRSS2 ${ }^{+}$ cell fractions, with all the signature and mixture matrices listed in Table S7. Compared to HBV- and HCV-infected livers, MAFLD livers had much higher TMPRSS2 ${ }^{+}$progenitor cells (Fig. 2f) indicating that MAFLD livers might be more susceptible to the SARS-CoV-2 virus. Similarly, the cirrhotic livers also had higher TMPRSS2 ${ }^{+}$progenitor cells than healthy livers, which was comparable to the scRNAseq results.

\section{Hypertension may increase the risk of liver injury for patients without pre-existing CLD}

A logistical regression model was used to identify the clinical characteristics, comorbidities, and symptoms that could increase the risk of liver injury among patients without preexisting CLD. As shown in Fig. S5, male sex was highly associated with the risk of liver injury suggesting that male patients are more likely to develop liver injury (see Table S5 for details). Furthermore, the association of hypertension and liver injury was significant for patients without preexisting CLD but not for patients with pre-existing CLD (Table S6).

\section{Risk scoring model for assessing liver injury and clinical outcomes for COVID-19 patients}

We built a risk scoring system based on 22 routine laboratory tests performed within 3 days after admission, such as liver function and routine blood tests. This system was used to evaluate the risk of liver injury in patients with COVID-19 as early as possible and provide guidance for the management of these patients. First, the univariate logistic regression model was applied to identify potential laboratory parameters with liver injury, and only those with an OR $>1$ and $p$ value $<0.001$ were retained. Next, the multivariate logistic regression models were used to determine the effect of those factors identified from the univariate logistic regression analysis. Finally, we identified 3 indicators at admission, including ALT (OR 1.07, 95\% CI 1.02-1.12), CRP (OR 1.02, 95\% CI 1.01-1.04), and LDH (OR 1.29, 95\% CI 1.20-1.39). Figure 3a showed the distribution of tested values for selected indicators. To determine the robustness of this model, a fivefold cross-validation method was employed. In this procedure, the original training data set is randomly partitioned into five subsets with the same sample size, and each subset is called one fold. 4 subsets are cross-validation training set, and 1 subset is cross-validation testing set. We trained our model on the cross-validation training set and test the model's predictions against the validation set. The average AUC of fivefold cross-validation was 85\% (Fig. 3c). Similarly, we selected 6 indicators (Fig. 3b) to predict whether patients would proceed to six-category scale scores of 5 or 6 . The average AUC reached 92\% (Fig. 3d). Since patients with pre-existing diseases including FLD, cirrhosis and hypertension were shown to be associated with higher risks, we also added these variables in our model, but the average AUC was not increased, being $85 \%$ and $91 \%$, respectively (Fig. S6). Therefore, we did not include the medical history in our final models. An R package provides all operations required for the clinical outcome prediction of new patients (https://github.com/liangyuan-njmu/Predi ctModel).

\section{Discussion}

Liver dysfunction has frequently been observed in patients with COVID-19 [16] who require intensive care [17]. We found that patients who developed liver injuries during hospitalization had higher mortality and ICU admission rates than those without liver injury and with liver injury upon admission. In addition, the patients with post-admission liver damage had significantly prolonged hospital stays.

No significant differences in mortality or ICU admission rates between patients with and without CLD were observed, suggesting that liver injury but not CLD is associated with disease severity and clinical outcomes in patients with COVID-19. 30.58\% of patients with FLD developed liver injury, which was higher than the overall percentage of liver injury in this COVID-19 cohort (17.9\%); this result suggests that patients with FLD may be at a higher risk of liver injury. MAFLD is strongly associated with obesity, and one recent study showed that COVID-19 more severely affected younger adults with obesity [18]. Given the known association between obesity and MAFLD [19], our observation of higher risks for patients with FLD and the higher abundance of $T M P R S S 2^{+}$progenitor cells in MAFLD livers may provide a possible explanation for why obese patients suffer more from COVID-19.

Both proteomics and transcriptomics data confirmed that small population cells in liver tissues were ACE2 positive [20], causing livers susceptible to SARS-CoV-2. Fan et al. and Lin et al. revealed that SARS-CoV-2 could directly bind to $A C E 2^{+}$cholangiocytes and damage bile duct tissue, suggesting a possible mechanism for SARSCoV-2-induced liver injury $[21,22]$. A more recent study found that ACE2 and TMPRSS2 are expressed in $T R O P 2^{+}$liver progenitor cells, a cholangiocyte-biased progenitor subpopulation, highlighting another potential cause of liver damage [23]. ACE2 has also been shown to be up-regulated in cirrhotic livers [24], indicating that patients with pre-existing cirrhosis may suffer from severe liver injury and faster disease progression. In this study, the six-category scale scores for patients with cirrhosis were higher. By analyzing public scRNA-seq data, we 

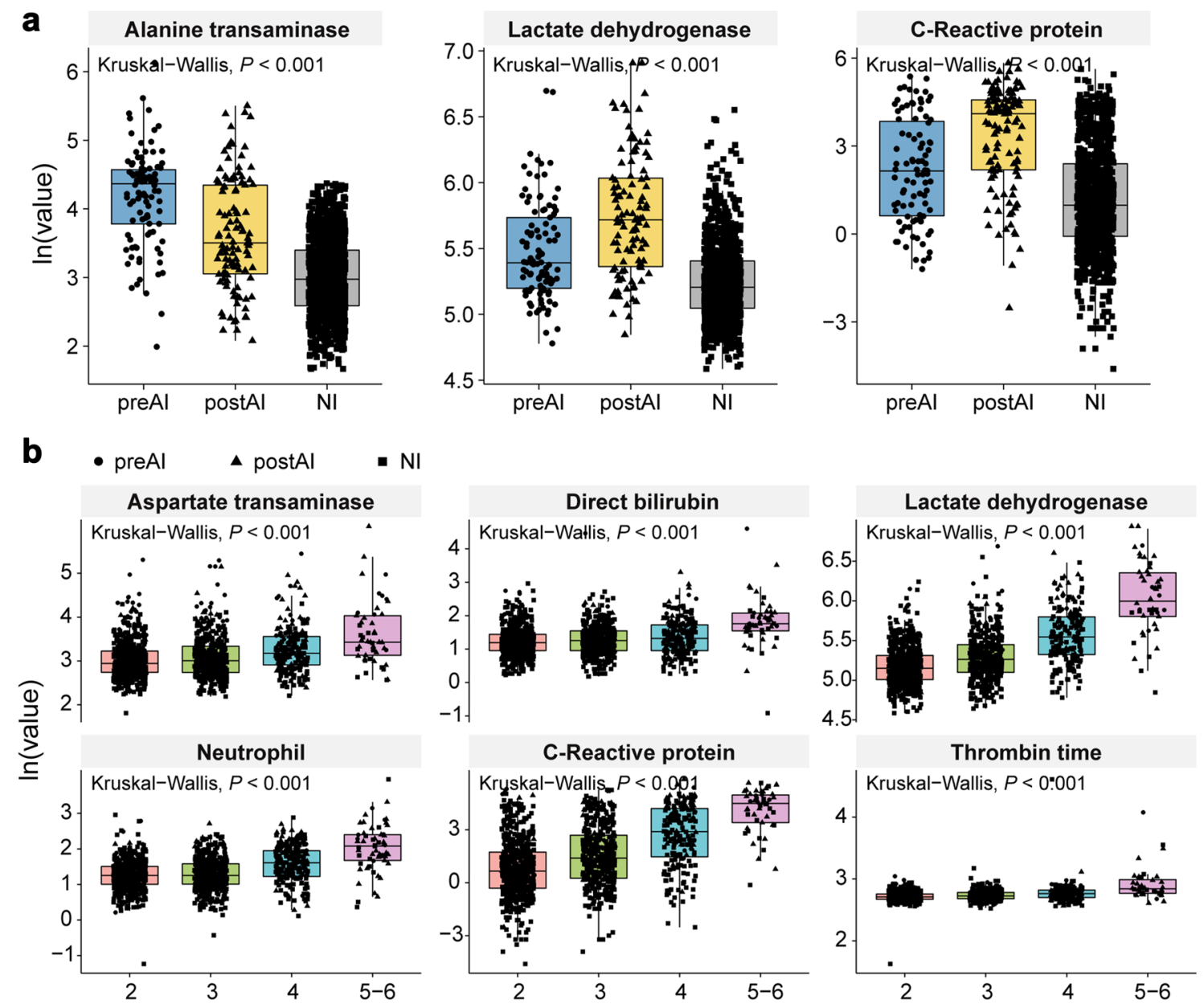

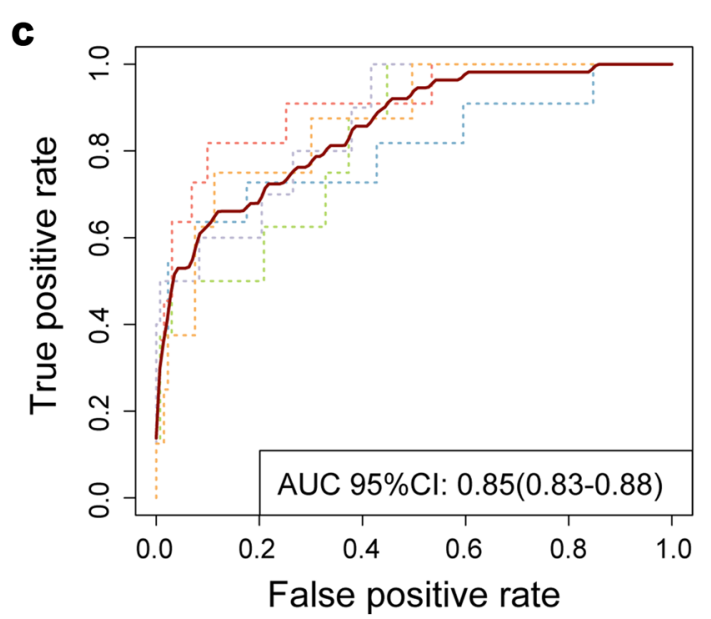

Fig. 3 Risk scoring model. a Boxplot for blood test results used in liver injury prediction model. The $p$ values were from Wilcoxon test. b Boxplot for blood test results used in prognosis prediction model. The $p$ values were from Kruskal-Wallis test. c The ROC curves of

revealed that the cirrhotic livers generated more $A C E 2^{+}$ and $T_{M P R S S}{ }^{+}$cells than healthy livers, and HBVinfected livers had the fewest $A C E 2^{+}$and TMPRSS2 ${ }^{+}$

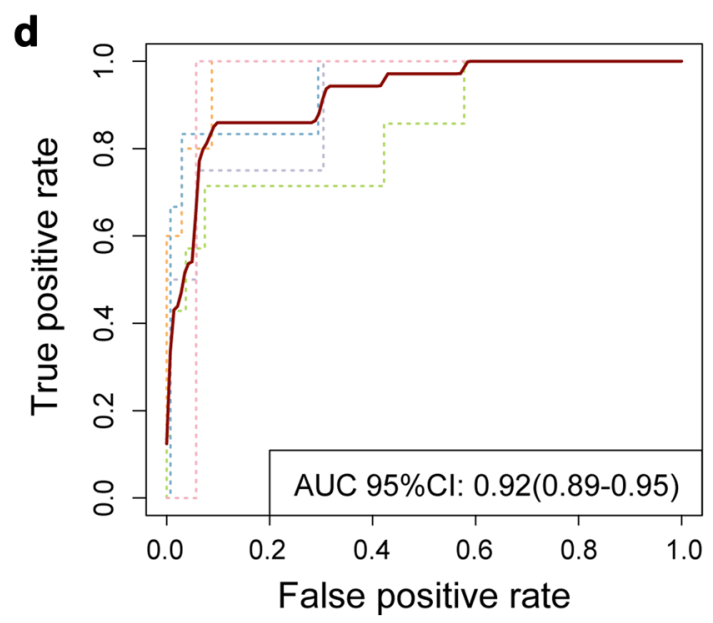

fivefold cross-validation for predicting liver injury during hospitalization. d The ROC curves of fivefold cross-validation for predicting prognosis

cells among the 3 liver types. CIBERSORTx estimation from bulk RNA-seq also confirmed that there were slightly more TMPRSS2 ${ }^{+}$progenitor cells in cirrhotic livers than 
HBV-infected and healthy livers. This may explain why patients with COVID-19 and cirrhosis had worse clinical outcomes than those with viral hepatitis.

Patient risk must be classified upon admission. Inspired by the MELD score, which is an existing scoring system used to prioritize liver transplantation and predict overall and postoperative outcomes in patients with hepatic and renal dysfunction [25-27], we constructed a similar scoring system to evaluate the liver impairment of patients with severe or critical COVID-19. The levels of ALT, lactate dehydrogenase, and CRP upon admission were used to build a linear regression equation to predict liver injury in subsequent hospital stays that could be used by clinicians to determine whether early liver protection management is required. Our other model can be used to predict those patients with COVID-19 who may have the highest severity of symptoms.

In conclusion, we comprehensively evaluated the clinical characteristics and laboratory parameters of patients with severe or critical COVID-19 symptoms. Patients who developed liver injuries during hospitalization had worse clinical outcomes and longer hospital stays. Our study suggests that performing liver protection treatments within one week of admission is beneficial for these patients. In particular, careful attention should be paid to patients with pre-existing CLD, cirrhosis, or FLD because of their worse liver function. Similarly, the liver function of patients with hypertension but without pre-existing CLD should be monitored. Further, we built a risk scoring system to predict liver injury upon admission. To conclude, we assessed the implication of liver injury and CLD for risk-stratification and management of patients with COVID-19, and we believe that our findings will help to improve clinical outcomes for these patients.

Acknowledgements This study was supported by grants from the National Natural Science Foundation of China (81971495, 81571564, 91442117, 81572893, 81972358, 91959113), CAMS Innovation Fund for Medical Sciences (No.2019-I2M-5-035), the National Science Foundation of Jiangsu Province (BRA2017533, BK20191490 and BE2016766), the 863 Young Scientists Special Fund (SS2015AA0209322), the Foundation of Jiangsu Collaborative Innovation Center of Biomedical Functional Materials, Key Foundation of Wuhan Huoshenshan Hospital (Grant No. 2020[18]), Key Research\& Development Program of Jiangsu Province (Grant Nos. BE2017733, BE2018713), Medical Innovation Project of Logistics Service (Grant No. 18JS005) and Basic Research Program of Jiangsu Province (Grant No. BK20180036).

\section{Compliance with ethical standards}

Conflict of interest We declare that we have no conflicts of interest.

Ethical approval It was a retrospective cohort study, approved by institutional ethics boards.

\section{References}

1. Hoffmann M, Kleine-Weber H, Schroeder S, Kruger N, Herrler T, Erichsen S, Schiergens TS, et al. SARS-CoV-2 cell entry depends on ACE2 and TMPRSS2 and is blocked by a clinically proven protease inhibitor. Cell. 2020;181(271-280):e278.

2. Huang C, Wang Y, Li X, Ren L, Zhao J, Hu Y, Zhang L, et al. Clinical features of patients infected with 2019 novel coronavirus in Wuhan, China. Lancet. 2020;395:497-506.

3. Cai Q, Huang D, Yu H, Zhu Z, Xia Z, Su Y, Li Z, et al. COVID19: abnormal liver function tests. J Hepatol. 2020;73:566-74.

4. Zhang C, Shi L, Wang FS. Liver injury in COVID-19: management and challenges. Lancet GastroenterolHepatol. 2020;5:428-30.

5. Fu Y, Zhu R, Bai T, Han P, He Q, Jing M, Xiong X, et al. Clinical features of patients infected with coronavirus disease 2019 with elevated liver biochemistries: a multicenter, retrospective study. Hepatology. 2020.

6. Xu Z, Shi L, Wang Y, Zhang J, Huang L, Zhang C, Liu S, et al. Pathological findings of COVID-19 associated with acute respiratory distress syndrome. Lancet Respir Med. 2020;8:420-2.

7. Lei F, Liu YM, Zhou F, Qin JJ, Zhang P, Zhu L, Zhang XJ, et al. Longitudinal association between markers of liver injury and mortality in COVID-19 in China. Hepatology. 2020;72:389-98.

8. Moon AM, Singal AG, Tapper EB. Contemporary epidemiology of chronic liver disease and cirrhosis. Clin Gastroenterol Hepatol. 2020;18:2650-66.

9. Xu XW, Wu XX, Jiang XG, Xu KJ, Ying LJ, Ma CL, Li SB, et al. Clinical findings in a group of patients infected with the 2019 novel coronavirus (SARS-Cov-2) outside of Wuhan, China: retrospective case series. BMJ. 2020;368:m606.

10. Ji D, Qin E, Xu J, Zhang D, Cheng G, Wang Y, Lau G. Nonalcoholic fatty liver diseases in patients with COVID-19: a retrospective study. J Hepatol. 2020;73:451-3.

11. Ramachandran P, Dobie R, Wilson-Kanamori JR, Dora EF, Henderson BEP, Luu NT, Portman JR, et al. Resolving the fibrotic niche of human liver cirrhosis at single-cell level. Nature. 2019;575:512-8.

12. Bost P, Giladi A, Liu Y, Bendjelal Y, Xu G, David E, BlecherGonen R, et al. Host-viral infection maps reveal signatures of severe COVID-19 patients. Cell. 2020;181(1475-1488):e1412.

13. Satija R, Farrell JA, Gennert D, Schier AF, Regev A. Spatial reconstruction of single-cell gene expression data. Nat Biotechnol. 2015;33:495-502.

14. Aizarani N, Saviano A, Mailly L, Durand S, Herman JS, Pessaux $\mathrm{P}$, et al. A human liver cell atlas reveals heterogeneity and epithelial progenitors. Nature. 2019;572:199-204.

15. Newman AM, Steen CB, Liu CL, Gentles AJ, Chaudhuri AA, Scherer F, Khodadoust MS, et al. Determining cell type abundance and expression from bulk tissues with digital cytometry. Nat Biotechnol. 2019;37:773-82.

16. Bangash MN, Patel J, Parekh D. COVID-19 and the liver: little cause for concern. Lancet Gastroenterol Hepatol. 2020.

17. Boettler T, Newsome PN, Mondelli MU, Maticic M, Cordero E, Cornberg M, Berg T. Care of patients with liver disease during the COVID-19 pandemic: EASL-ESCMID position paper. JHEP Rep. 2020;2:100113.

18. Kass DA, Duggal P, Cingolani O. Obesity could shift severe COVID-19 disease to younger ages. Lancet. 2020;395:1544-5.

19. Polyzos SA, Kountouras J, Mantzoros CS. Obesity and nonalcoholic fatty liver disease: from pathophysiology to therapeutics. Metabolism. 2019;92:82-97.

20. Hikmet F, Mear L, Edvinsson A, Micke P, Uhlen M, Lindskog C. The protein expression profile of ACE2 in human tissues. MolSystBiol. 2020;16:e9610. 
21. Chai X, Hu L, Zhang Y, Han W, Lu Z, Ke A, Zhou J, et al. Specific ACE2 expression in cholangiocytes may cause liver damage after 2019-nCoV Infection. In: bioRxiv; 2020.

22. Zhao B, Ni C, Gao R, Wang Y, Yang L, Wei J, Lv T, et al. Recapitulation of SARS-CoV-2 infection and cholangiocyte damage with human liver ductal organoids. Protein Cell. 2020;11:771-5.

23. Seow JJW, Pai R, Mishra A, Shepherdson E, Lim TKH, Goh BKP, Chan JKY, et al. scRNA-seq reveals ACE2 and TMPRSS2 expression in TROP2+ Liver Progenitor Cells: Implications in COVID-19 associated Liver Dysfunction. bioRxiv. 2020. https:// doi.org/10.1101/2020.03.23.002832.

24. Herath CB, Warner FJ, Lubel JS, Dean RG, Jia Z, Lew RA, Smith AI, et al. Upregulation of hepatic angiotensin-converting enzyme 2 (ACE2) and angiotensin-(1-7) levels in experimental biliary fibrosis. J Hepatol. 2007;47:387-95.

25. Kamath PS, Wiesner RH, Malinchoc M, Kremers W, Therneau TM, Kosberg CL, D'Amico G, et al. A model to predict survival in patients with end-stage liver disease. Hepatology. 2001;33:464-70.

26. Malinchoc M, Kamath PS, Gordon FD, Peine CJ, Rank J, ter Borg PC. A model to predict poor survival in patients undergoing transjugular intrahepatic portosystemic shunts. Hepatology. 2000;31:864-71.

27. Kamath PS, Kim WR, Advanced Liver Disease Study G. The model for end-stage liver disease (MELD). Hepatology. 2007;45:797-805.

Publisher's Note Springer Nature remains neutral with regard to jurisdictional claims in published maps and institutional affiliations.

\section{Authors and Affiliations}

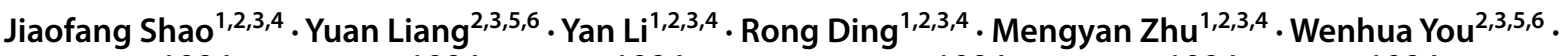 Ziyu Wang ${ }^{1,2,3,4} \cdot$ Bin Huang ${ }^{1,2,3,4} \cdot$ Min Wu ${ }^{1,2,3,4} \cdot$ Tingting Zhang ${ }^{1,2,3,4} \cdot$ Kening $\mathrm{Li}^{1,2,3,4} \cdot$ Wei $\mathrm{Wu}^{1,2,3,4}$.

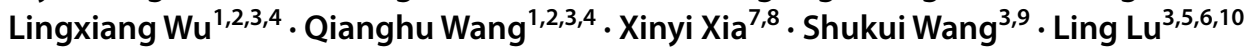

Jiaofang Shao

shaojf@njmu.edu.cn

Yuan Liang

liangyuan@njmu.edu.cn

Yan Li

liyan-icy@163.com

Rong Ding

dingrong@njmu.edu.cn

Mengyan Zhu

zhumengyan1994@njmu.edu.cn

Wenhua You

youwh@njmu.edu.cn

Ziyu Wang

wangziyu@njmu.edu.cn

Bin Huang

huangbin@njmu.edu.cn

Min Wu

biowumin@njmu.edu.cn

Tingting Zhang

zhangtingting@njmu.edu.cn

Kening Li

likeninghappy@163.com

Wei Wu

wuwei.bioinformatics@gmail.com

Lingxiang $\mathrm{Wu}$

woolingxiang@gmail.com
1 Research Center for Global Health, School of Public Health, Nanjing Medical University, Nanjing 211166, Jiangsu, China

2 Department of Bioinformatics, Nanjing Medical University, 101 Longmian Avenue, Jiangning District, Nanjing 211166, Jiangsu, China

3 Jiangsu Key Lab of Cancer Biomarkers, Prevention and Treatment, Collaborative Innovation Center for Personalized Cancer Medicine, Nanjing Medical University, Nanjing, China

4 Collaborative Innovation Center for Cardiovascular Disease Translational Medicine, Nanjing 211166, Jiangsu, China

5 The Affiliated Cancer Hospital and Hepatobiliary Center, The First Affiliated Hospital of Nanjing Medical University, 300 Guangzhou Road, Nanjing, China

6 Research Unit of Liver Transplantation and Transplant Immunology, Chinese Academy of Medical Sciences, Nanjing, China

7 COVID-19 Research Center, Institute of Laboratory Medicine, Jinling Hospital, Nanjing University School of Medicine, Nanjing Clinical College of Southern Medical University, Nanjing 210002, Jiangsu, China

8 Joint Expert Group for COVID-19, Department of Laboratory Medicine and Blood Transfusion, Wuhan Huoshenshan Hospital, Wuhan 430100, Hubei, China

9 Department of Laboratory Medicine, Nanjing First Hospital, Nanjing Medical University, Nanjing 210006, Jiangsu, China

10 State Key Laboratory of Reproductive Medicine, Nanjing Medical University, Nanjing, China 